\title{
An Improved F-Tablet for Handwriting Signal Capture
}

\author{
Ping Fang ${ }^{1,2}$, ZhongCheng $\mathrm{Wu}^{1}$, Ming Meng ${ }^{1,2}$ and YunJian $\mathrm{Ge}^{1}$ \\ 1.State Key Laboratories of Transducers Technology \\ Institute of Intelligent Machines, CAS \\ Hefei, Anhui Province, China 230031 \\ 2. Department of Automation \\ University of Science \& Technology of China \\ Hefei, Anhui Province, China \\ pingfang@ustc.edu, zcwu@iim.ac.cn,emnming@yahoo.com.cn,yjge@iim.ac.cn,
}

\begin{abstract}
At present, many researches have been done on the handwriting using static or dynamic signals. And we have devised a force sensitive tablet named F-Tablet, which is capable of capturing both the dynamic handwriting information and the static trajectory of the writing pen. With the core part of the multi-dimension force/torque sensor, the tablet can capture the two torques and three forces directly. And with the specially designed structure, other dynamic and static information can be got indirectly. Improvement has been made to the original design to get better performance. Ordinary pens can be used to write on the tablet. The tablet can also be used as an input device for computers or as a development tool for children to improve handwriting skills.
\end{abstract}

Index Terms - F-Tablet, handwriting signal, multi- dimension force/torque sensor.

\section{INTRODUCTION}

Biometrics has become a hot subject in recent years because the need to prevent unauthorized access to all kinds of e-data during this information age. Data from physical biometrics such as fingerprints, hand geometry and iris scans, behavioral biometrics such as signature, voiceprint, gait and mannerisms, and chemical biometrics such as DNA and body odor have bas been acquired and analyzed for security applications.

During writing, a writer writes not only the context, but also his identity, which is implied in the dynamic writing process and the static handwriting. And because of the long history of signature and the convenience of the pen and papers, we are all accustomed to having a signature signed on file to be used as a basis of comparison for verifying our signature [1]. Many researches have been done on signature and some applications have been found. Rejean Plamondon has studied the models and the dynamic characters of writing; Sargur N. Srihari has done much work on the static handwriting information. The work of Sung-Hyuk Cha.K deals with the establishing handwriting individuality. The Name and Address Block Reader (NABR) developed at CEDAR was installed at most United States IRS processing centers, beginning in 1995. Cyber-SIGN provides products for signature verification by analyzing the shape, speed, stroke, pressure and timing information during the act of signing. Communication Intelligence Corporation has developed transaction and communication enabling technologies for electronic signatures, handwritten biometric signature verification, data security, and data compression. Softpro SignPlus, Valyd, WonderNet, LCI SMARTpen and some other companies have all provide solutions for signature identification/verification.

Generally, the signature can be studied using the dynamic handwriting signals, static handwriting signals or both the dynamic signals and the static signals at the same time. As the dynamic signals are apparently much harder to be imitated by others than the static signals, so a wide variety of devices have been devised for capturing the dynamic handwriting signals. An accelerometer pen is used to capture the two direction accelerations and pressure in the z-axis [2]. A dual axis accelerometer is used to get the signature's dynamics [3]. The SmartPen is used to capture forces on the pen-tip in three directions and angles of the pen-shaft in two dimensions (relative to the writing surface) [4]. A "digital pen" is used for detecting $\mathrm{z}$-axis pressure [5].

As we can see that the devices designed for capture dynamic handwriting signals can only get the pressure or can only get the forces with no other handwriting signals. This paper introduces a novel multi-dimension force sensitive tablet for handwriting signal capture based on USB, which is named F-Tablet. This device is capable of capturing both the dynamic and static handwriting information. First, the three perpendicular forces between the writing pen and the writing tablet directly are captured with a multi-dimension force/torque sensor. Then, the other handwriting signals such as trajectory, velocities and accelerations of the writing pen can also be calculated indirectly.

The work here is mainly based on the work of [6]. The problem of the F-Tablet is analyzed and improvements are made to the structure to get better performance. The software is also modified to show the forces and trajectories of the writing. An USB interface and some filtering circuit are added to the conditioning circuit. In the next chapter, the structure and working principles of the F-Tablet for handwriting signal capture are described at first. Then the signal conditioning circuit is given in the chapter followed. The experimental results are presented and discussed in chapter 4 . The problem and improvements are analyzed in chapter 5 . And conclusions are made in the last chapter.

\section{THE F-TABLET}

This F-Tablet is capable of capture three perpendicular forces of the pen-tip to the contacting plane and torques in two directions directly with a multi-dimension force/torque sensor. With the specially designed structure, the other dynamic signals and the static trajectory of the pen-tip can also be calculated indirectly. 
Proceedings of 2004 International Conference on Information Acquisition
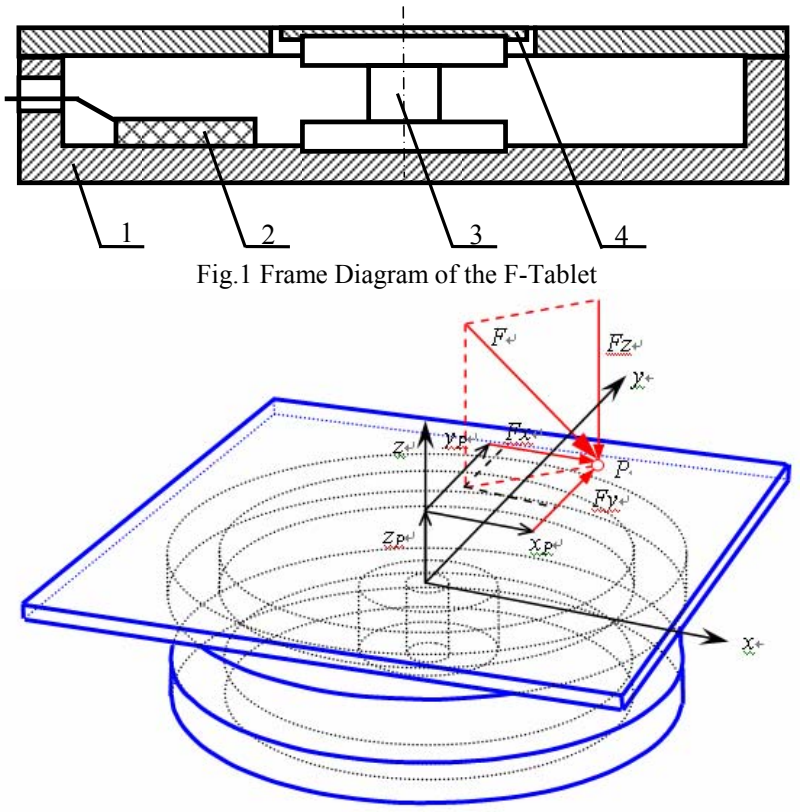

Fig.2 Schematic Diagram of Force Action

Fig.1 shows the frame diagram of the F-Tablet. It consists of 4 parts. Part 1 is a cuboid box and wraps all other parts. The signal conditioning circuit (part 2) processes the signals from the 3rd part, the multi-dimension force/torque sensor. Part 4 is the input tablet of $60 \times 60 \mathrm{~mm}^{2}$, on which pens write. It is assembled together with the top of the sensor and there is a small clearance to the wrap box.

The multi-dimension force/torque sensor, of which the bottom is assembled into the wrap box, is the core part of this F-Tablet. It measures the forces and torques of the pen-tip. The details of the sensor's working principle can be found in [7].

The working principle can be learned from Fig.2. When a pen writes on the input tablet at point $\mathrm{P}$, force $\mathrm{F}$ can be decomposed into forces in three perpendicular directions, $F_{x}\left(t_{i}\right), F_{y}\left(t_{i}\right), F_{z}\left(t_{i}\right)$. At the same time, $M_{x}\left(t_{i}\right)$ and $M_{y}\left(t_{i}\right)$ are exerted on the input tablet. These five force elements are all functions of time $t_{i}$ and can be measured directly by the multidimension force/torque sensor. The coordinates of the point $\mathrm{P}$ can be calculated from the equilibrium of moments. They can be expressed as (1):

$$
\left\{\begin{array}{l}
x_{p}\left(\mathrm{t}_{\mathrm{i}}\right)=\frac{M_{y}\left(\mathrm{t}_{\mathrm{i}}\right)-F_{x}\left(\mathrm{t}_{\mathrm{i}}\right) \cdot h}{F_{z}\left(\mathrm{t}_{\mathrm{i}}\right)} \\
y_{p}\left(\mathrm{t}_{\mathrm{i}}\right)=\frac{-M_{x}\left(\mathrm{t}_{\mathrm{i}}\right)-F_{y}\left(\mathrm{t}_{\mathrm{i}}\right) \cdot h}{F_{z}\left(\mathrm{t}_{\mathrm{i}}\right)}
\end{array}\right.
$$

where $h$ denotes the distance between he input tablet and the origin of the coordinate, that is $z_{p} ; i=0,1,2, \ldots$. The velocities and accelerations are the derivative of the two coordinates, which can be expressed as (2) and (3).

$$
\begin{aligned}
& \left\{\begin{array}{l}
v_{x}\left(t_{j}\right)=\frac{x\left(t_{j}\right)-x\left(t_{j-1}\right)}{\Delta t} \\
v_{y}\left(t_{j}\right)=\frac{y\left(t_{j}\right)-y\left(t_{j-1}\right)}{\Delta t}
\end{array}\right. \\
& \left\{\begin{array}{l}
a_{x}\left(t_{k}\right)=\frac{v_{x}\left(t_{k}\right)-v_{x}\left(t_{k-1}\right)}{\Delta t} \\
a_{y}\left(t_{k}\right)=\frac{v_{y}\left(t_{k}\right)-v_{y}\left(t_{k-1}\right)}{\Delta t}
\end{array}\right.
\end{aligned}
$$

where $v_{x}, v_{y}$ and $a_{x}, a_{y}$ are the velocities and accelerations in $\mathrm{x}$ and $\mathrm{y}$ direction respectively; $\mathrm{i}=1,2,3, \ldots ; \mathrm{k}=2,3,4, \ldots$. When $\Delta t$ comes to zero, we can get the instantaneous velocities and accelerations. Generally speaking, $\Delta t$ can't be too small. But when $\Delta t$ is small enough, the velocities and accelerations calculated can be viewed as the instantaneous ones.

The angle between the writing pen and the input tablet can be calculated from the three perpendicular forces. With the coordinates we can figure out the static image of the handwriting. Even the stroke order can be got by analysing the three perpendicular forces.

From the analysis above, we can see that both the dynamic and static handwriting information can be got with the force sensitive device.

\section{CONDITIONING CIRCUIT}

The upper limit of the frequency of handwriting is about $20 \mathrm{~Hz}$ [2]. Results from our research shows that the maxim writing pressure is about $5 \mathrm{~N}$, the normal writing pressure is between $1 \sim 3 \mathrm{~N}$ and that varies with different person. This FTablet is designed to measure forces between $0 \sim 10 \mathrm{~N}$, and the system resonance frequency is $140 \mathrm{~Hz}$, so the tablet is capable of capture the dynamic handwriting signal without distortion. The conditioning circuit as Fig. 3 shows is designed to get the 5 channel output from the multi-dimension sensor. As the 5 channel have the same structure, only the 5th channel is presented in details.

First the 5 output signals from the Wheatstone bridge are amplified to big enough to be sampled. As the 5 channel have different measurement range, we adjust the operational amplifier suitably so that the maxim force or torque doesn't cause any saturation and the small signals can be observed at the same time. After that, a RC filtering circuit is used so that only signals with frequency between 0 and $20 \mathrm{~Hz}$ can get across. A 12 bit $\mathrm{AD}$ is decided to do the conversion. And every channel will be sampled at the frequency of $100 \mathrm{~Hz}$.

Fz can be decomposed into three parts, the gravitation of the input tablet, the force exerted to the input tablet when writing and the fluctuation of the offset value with the time. The other forces and torques don't have the gravitation of the input tablet. During calibration, the effect of the input tablet will be eliminated. And the offset value can be viewed as a constant during writing and can be subtracted from the 
captured value at the beginning of writing with specially designed protocol.

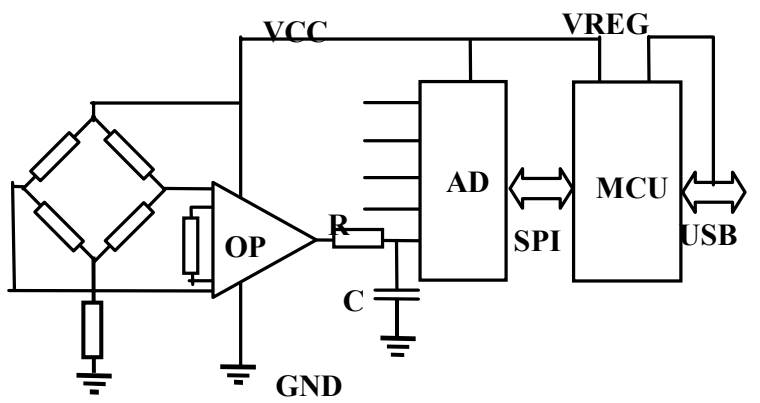

Fig.3 Diagram of the Conditioning Circuit

USB has many advantages such as self-identifying peripherals, automatic mapping of function to driver and configuration, dynamically attachable and reconfigurable peripherals. USB as a new interface standard with a long term perspective is preferred external connection to a host PC. And the calculation results show that the whole conditioning circuit can be driven by the power from the USB interface. USB interface is added to the conditioning circuit. MCU get power from USB and regulates it to power other devices. The USB is sure to give users more convenience.

\section{EXPERIMENTAL RESULTS}

This experiment is designed to verify whether the novel writing tablet is capable of capture both the dynamic and the static handwriting information. Because the forces and torques are measured by the tablet, so there is no requirement on the writing pen. An ordinary pen is used to write a Chinese character on the input tablet. The conditioning circuit gets the 5 channel signals and transmits them to the host PC through USB interface. Test software is developed to display the trajectory using (1) and draw the three forces at the same time. The width of the stroke is decided by the pressure between the pen-tip and the input tablet or Fz. And because the coordinates of the trajectory are calculated from Fx, Fy, $\mathrm{Fz}, \mathrm{Mx}$ and $\mathrm{My}$, so the shape of the displayed character implies all the forces and torques of the writing.

Fig. 4 and Fig. 5 show the shape of the Chinese character and the three forces during writing. From the shape of the character, we can see that not only the trajectory of the pen, but also the writing style of the writer can be recorded. And from the force curve and the static shape we can even get the stroke order. The earliest data captured is displayed on the right side in Fig.5. Fz is translated to be positive to give a good view. How the pen has gone can be figured out combining Fx with Fy. First, the pen wrote the top point. Then it went from left to right. Then it goes from top right to down left. And then wrote the last stroke.

The velocities and accelerations are calculated with (2) and (3) respectively. Other handwriting information such as the angle between the writing pen and the input tablet can also be calculated from the three perpendicular forces. Fig.6 shows the velocity in $\mathrm{X}$ direction drawn with MATLAB.
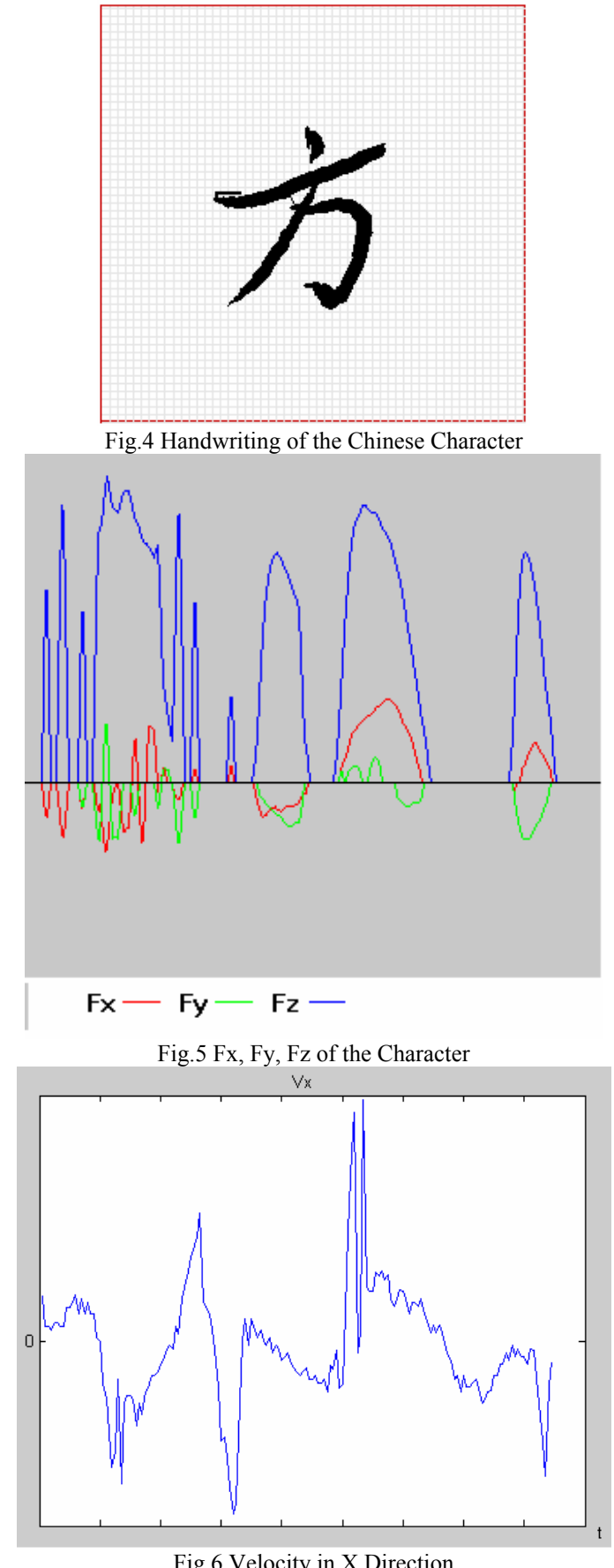

\section{PROBLEM ANALYSIS AND IMPROVEMENT METHODS}

This F-Tablet can capture the three perpendicular forces between the writing pen and input tablet accurately. But the accuracy of trajectory calculated from the forces and torques has much space to be improved due to the constructive parameters such as stiffness of the input tablet. The error of the trajectory depends on the position of the pen-tip and increases as the pen-tip moves to the border of the input tablet 
[7]. And the angle of the force vector, that is the angle between the input tablet and the writing pen, also affect the resolution of the trajectory [8]. The resolution of the trajectory can be expressed as:

$$
\left\{\begin{array}{l}
d x=\frac{-F_{z} d M_{y}+F_{z} h d F_{x}-\left(-M_{y}+F_{x} h\right) d F_{z}}{F_{z}^{2}} \\
d y=\frac{F_{z} d M_{x}+F_{z} h d F_{y}-\left(M_{x}+F_{y} h\right) d F_{z}}{F_{z}^{2}}
\end{array}\right.
$$

We can see that $d x$ and dy are very sensible to Fz. Both the pen-down and pen-up will give impacts to the system, which will affect the static handwriting shape. This can be found very clear in Fig.4.

As part 4 of this F-Tablet is just a stiff tablet on which pens write. We can substitute it with a pressure sensitive tablet, which can be used to capture the trajectory of the writing point with very high resolution. And the pressure sensitive tablet can also provide the signal that whether the pen is writing, with which we can filter the impact and get better results.

\section{CONCLUSION AND FUTURE WORK}

In this paper, a novel multi-dimension force sensitive tablet named F-Tablet for handwriting signal capture based on USB is introduced. With a special multi-dimension force/torque sensor, we can get the three perpendicular forces of the pen-tip to the contacting plane and torques in two directions directly. The trajectory, velocities, accelerations and angle of the pen-tip can be calculated from the directly measured results. And there is no special requirement on the writing pen. This device could also be used as a new writing input tool for computers. With well developed software, this F-Tablet can even be used as a development tool for children to improve their writing skills.
A number of experiments have been conducted using this F-Tablet. Experimental results show that the tablet is indeed capable of obtaining forces, trajectory, velocities and acceleration signals of the pen-tip. The further work is to do some research work on writer identification/verification using one kind or several kinds of the signals captured with the tablet.

\section{ACKNOWLEDGMENT}

The authors want to thank Prof. Xu D.Z and Mrs Sheng Fei for their support to this work.

\section{REFERENCES}

[1] R. Plamondon, and S.N. Srihari, "On-line and off-line handwriting recognition: a comprehensive survey," IEEE Trans. on Pattern Analysis and Machine and Machine Intelligence, vol.22, pp. 63-85, 2000.

[2] R. Baron and R Plamondon, "Acceleration Measurement with an Instrumented Pen for Signature Verification and Handwriting Analysis," IEEE Transactions on Instrumentation and Measurement, Vol. 38, pp. 1132-1138, 1989.

[3] J. Martinez, J. de J. Lopez, and F. Luna Rosas, "A low-cost system for signature recognition," 42nd Midwest Symposium on Circuits and Systems, vol. 1, pp. 101-104, 1999.

[4] R. Martens and L. Claesen, "Incorporating local consistency information into the online signature verification process," International Journal on Document Analysis and Recognition, vol. 1, pp. 110-115, 1998.

[5] K. Tanabe, M. Yoshihara, H. Kameya and S. Mori, "Automatic Signature Verification Based on the Dynamic Feature of Pressure," Sixth International Conference on Document Analysis and Recognition, Proceedings, pp. 1045-1049, 2001.

[6] Ping Fang, ZhongCheng Wu and Ming Meng, "A Novel Tablet for OnLine Handwriting Signal Capture", The 5th World Congress on Intelligent Control and Automation, 2004, in press.

[7] M. F. Bobbert and H. C. Schamhardt, "Accuracy of determining the point of force application with piezoelectric force plates," J. Biomechan., vol. 23 , no. 7 , pp. 705-710, 1990

[8] Heinz-Bodo Schmiedmayer and Josef Kastner, "Parameters influencing the accuracy of the point of force application determined with piezoelectric force plates", Journal of Biomechanics 32, pp1237-1242, 1999. 